\title{
Tensor-Based Shot Boundary Detection in Video Streams
}

\author{
Bogusław Cyganek ${ }^{1}$ (D) Michał Woźniak ${ }^{2}$
}

Received: 21 April 2017/Accepted: 28 June 2017/Published online: 8 August 2017

(C) The Author(s) 2017. This article is an open access publication

\begin{abstract}
This paper presents a method for content change detection in multidimensional video signals. Video frames are represented as tensors of order consistent with signal dimensions. The method operates on unprocessed signals and no special feature extraction is assumed. The dynamic tensor analysis method is used to build a tensor model from the stream. Each new datum in the stream is then compared to the model with the proposed concept drift detector. If it fits, then a model is updated. Otherwise, a model is rebuilt, starting from that datum, and the signal shot is recorded. The proposed fast tensor decomposition algorithm allows efficient operation compared to the standard tensor decomposition method. Experimental results show many useful properties of the method, as well as its potential further extensions and applications.
\end{abstract}

Keywords Video shot detection - Anomaly detection - Tensor decomposition · Tensor frames · Dynamic tensor analysis

\section{Introduction}

Enormously increasing amounts of visual information raise the needs for the development of automatic data analysis methods. Among these, special attention is paid to the video summarization methods. Their goal is to give a user a short and useful visual abstract of the entire video sequence. This can be further used to

Bogusław Cyganek

cyganek@agh.edu.pl

Michał Woźniak

Michal.Wozniak@pwr.wroc.pl

1 AGH University of Science and Technology, Al. Mickiewicza 30, 30-059 Kraków, Poland

2 Wrocław University of Science and Technology, Wybrzeże Wyspiańskiego 27, 50-370 Kraków, Poland 
improve efficacy of video cataloging, indexing, archiving, information search, data compression, to name a few [47]. These methods, in turn, rely on efficient algorithms of boundary detection in the visual streams. Basically, the summarization methods are divided into static and dynamic ones [33, 35]. In the former, a set of consecutive frames with sufficiently coherent contents is represented by a single representative frame, called a key frame. On the other hand, a dynamic summarization, also called a video skim, relies on selecting the most relevant small fractions of the video sequences [19]. This allows for better representation of the concise portions of the video, thanks to inclusion of visual and audio tracks. A key step in these methods is to track a video stream and recognize regions of sufficiently abrupt change in the visual content. However, such generally stated task is subjective and depends on specific video content which manifests with different segmentations done in human-made experiments. For the purpose of automatic video summarization, a significant research is conducted toward the development of methods for computation of sufficiently discriminative features [20, 45]. In this spirit, majority of the methods first employ extraction of specific features, which are then used for video clusterization and classification.

Our proposed approach to video analysis significantly differs from the featurebased methods. Most of all we treat the frames holistically as 2D or 3D tensorstherefore, we call them tensor frames. This way, considering time dimension, the whole video can be represented as a $4 \mathrm{D}$ tensor. Such a framework allows easy extensions to higher dimensions, if necessary. Further on, we do not extract any features from the frames which is a common practice in majority of the video summarization methods proposed in the literature. In addition, in many of the available works on video segmentation, temporal analysis is not fully exploited. However, this is a very important factor, since the consecutive frames show high correlation throughout a scene. On the other hand, our framework allows for a uniform approach to video structure analysis, both in spatial and temporal dimensions. Our method relies on the tensor stream processing concept proposed by Sun et al. [44]. However, in our approach, this method was improved to allow for efficient processing of the video streams, as will also be discussed. To the best of our knowledge, the tensor stream tensor analysis method has not been used in the problem of video summarization. Our contribution is as follows:

- the original tensor framework for video shot detection based on the tensor model and its continuous build-and-update mechanism;

- novel concept of drift detectors in tensor video streams based on statistical analysis of the differences of the tensor-frame projections onto the model;

- fast tensor subspace computation with the modified fixed-point eigenvalue method, endowed with a mechanism of dominant rank determination and reinitialization from previous eigenvectors.

As mentioned, the proposed method can operate with any dimensional signals, such as monochrome and color videos, as presented in the experimental section of this paper. No feature extraction is necessary and the signal is treated "as is". Therefore, the method can work with frames of any dimension and content, such as 
hyperspectral or compressed signals, etc. However, due to the so-called "curse of dimensionality" when going into higher dimensions, we face the problem of high memory and computational requirements $[4,55]$. In addition, if statistical properties of the processed signal are known, then feature-based methods can perform better taking advantage of the expert knowledge. Nonetheless, the proposed framework can also accept features and signal as its input, as will be discussed.

The rest of this paper is organized as follows. Section 2 outlines the basic concepts related to video signal representation and analysis, as well as presents related works in the area of the video segmentation, tensor processing, and data streams. A short overview on tensors and their decomposition is presented in Sect. 3. The main concepts of the proposed method are presented in Sect. 4. Specifically, system architecture and basic concepts of tensor stream analysis are presented in Sect. 4.1, and a modified best rank- $\left(R_{1}, R_{2}, \ldots, R_{P}\right)$ tensor decomposition for stream tensors is discussed in Sect. 4.2. Efficient computation of the dominating tensor subspace is outlined in Sect. 4.3. Computation of the proposed drift detection in video tensor streams is presented in Sect. 4.4. Finally, the video tensor stream model build-and-update scheme is presented in Sect. 4.5. Experimental results are presented in Sect. 5, while conclusions are presented in Sect. 6.

\section{Overview of Video Signal Representation and Analysis}

This section contains a brief introduction to the video representation and signal analysis methods for the purpose of scene shot detection and video summarization.

\section{Video Structure and Analysis}

A video is a multidimensional digital signal organized as a stream of still images, called frames, as depicted in Fig. 1. These may be monochrome or color images. Visual signals are accompanied by the audio signal, which is not considered in this work, though. As will be further discussed, frames as well as videos can be interpreted as tensors. In this framework, a monochrome frame is represented as a $2 \mathrm{D}$ tensor, a color frame as a 3D tensor, and so on. Thus, these are called tensor frames. Content of frames changes in time-giving impression of smooth motion to the observer. On the other hand, consecutive frames that contain similar content are grouped together and form the so-called shots. Finally, a set of consecutive shots with semantically similar content is called a scene. These are visualized in Fig. 1.

There can be different types of content change in video signals. These can be grouped as follows:

- Hard cuts - an abrupt change of a content;

- Soft cuts - a gradual change of a content.

The latter can further divided into the following categories:

- Fade-in/out — a new scene gradually appears/disappears from the current image;

- Dissolve-a current shot fades out, whereas the incoming one fades in. 


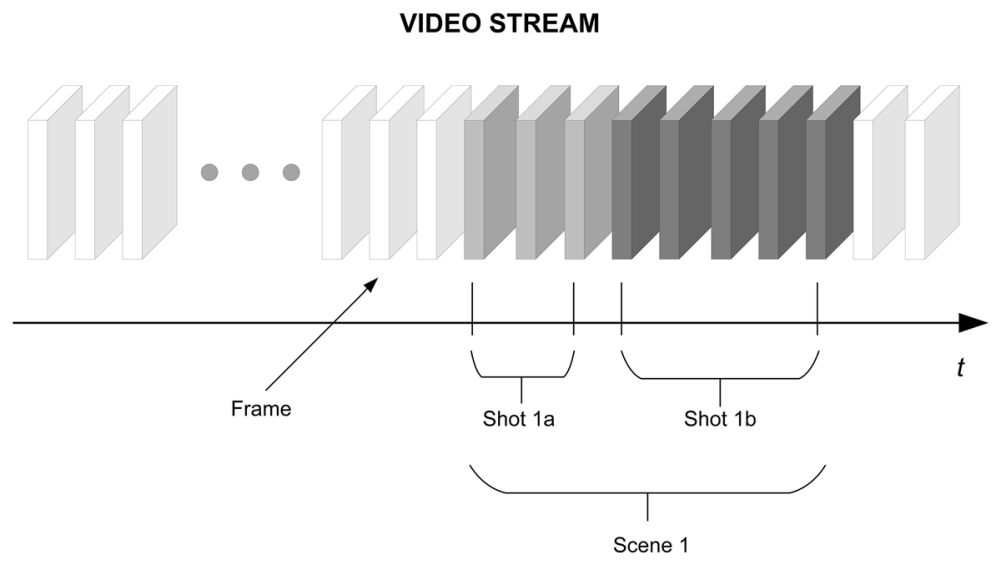

Fig. 1 Structure of a video stream with shown frames, shots, and scenes. Each frame is represented as a 2D or 3D tensor frame for monochrome and color versions, respectively

Figure 2 presents two different types of exemplary shots excerpted from the videos from the Open-Video database [21]. The first one, presented on top of Fig. 2, is a hard shot in the Drift Ice sequence. On the other hand, the bottom row in Fig. 2 depicts a soft shot from The Great Web of Water video.

Detection of different types of shots is a special case of the concept drift detection in streams of data [17, 28, 41]. In the case of video signals, analyzed in this paper, detection of different types of shots requires development of special methods which account for different statistical properties of the video signals. This problem, as well as the proposed solutions, is discussed in further sections of this paper.

\section{Related Works}

As already mentioned, due to large social expectations, video analysis and especially video summarization gain high attention in research community.
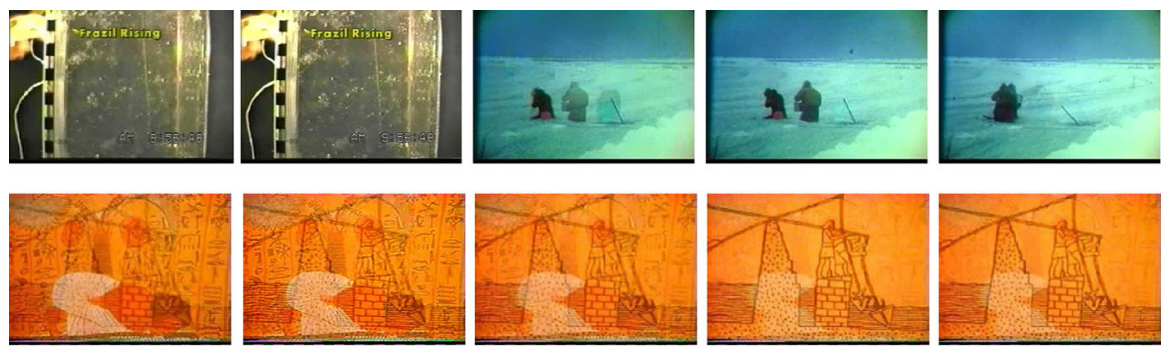

Fig. 2 Examples of different shots in video signals. Top a hard shot in the Drift Ice sequence (frames 752-756). Bottom a soft shot from The Great Web of Water (odd frames 931-939). The images from the open-video site [21] 
Recently, many automatic systems for video segmentation have been proposed. A prevailing majority of them relies on specific feature extraction and further clusterization and classification [10, 40,54]. A survey of video indexing, including methods of temporal video segmentation and keyframe detection, is contained in the paper by Asghar et al. [1]. Description of the main tasks in video abstraction is provided in the paper by Truong and Venkatesh [45]. On the other hand, Fu et al. present an overview of the multiview video summarization methods [15]. Valdes and Martinez discuss on efficient video summarization and retrieval tools [47]. A recent survey of video scene detection methods is provided in the paper by Fabro and Böszörmenyi [14]. They classify the video segmentation methods into seven groups, depending on the low-level features used for the segmentation. In result, the visual-based, audio-based, text-based, audio-visual, visual-textual, audio-textual, and hybrid segmentations can be listed. An overview of papers, presenting more specific methods, is as follows.

Lee et al. propose a unified scheme of shot boundary and anchor shot detection in news videos [33]. Their method relies on a singular value decomposition and the socalled Kernel-ART method. The anchor shot detection is based on the skin color detector, face detector, and support vector data descriptions with non-negative matrix factorization. However, their method is limited to the videos containing specific scenes, persons, etc. On the other hand, DeMenthon et al. presented a simple system of video summarization by curve simplification [37]. In their approach, a video sequence is represented as a trajectory curve in a highdimensional feature space. It is then analyzed thanks to the proposed binary curvesplitting algorithm. This way, partitioned video is represented with a tree-like structure. However, the method relies on feature extraction from the video. These are DC coefficients used in the MPEG-coding standard. The other method of video summarization was proposed by Mundur et al. [38]. In their method, keyframebased video summarization is computed with the Delaunay clustering [29]. In the STIMO system proposed by Furini, a method for moving video storyboard for the web scenario is proposed [16]. Their method is optimized for Web operation to produce on-the-fly video storyboards. The method is based on a fast-clustering algorithm that selects the most representative video content using color distribution in the HSV color space, computed on a frame-by-frame basis.

A method, called VSUMM, is proposed in the paper by de Avila et al. [2]. Their approach is based on computation of color histograms from frames acquired from a video stream one per second, then clustered with the k-means method. Further on, for each cluster, a frame closest to the cluster center is chosen as the so-called keyframe that represents a given slice of a video. De Avila et al. also developed a method of video static summaries evaluation which is used in further works for comparison. A significant contribution of their work is that all of the user annotations used in their experiments were also made available from the Web, what greatly facilitates further comparative evaluations [22]. In addition, in this paper, we refer to their data and provide comparative results, as will be discussed.

Color histogram for video summarization is also used in the method proposed by Cayllahua-Cahuina et al. [7]. In their method, at first, a 3D histogram of $16 \times 16 \times 16$ bins is calculated directly in the RGB color space. This way, 
obtained vectors of 4096 elements are further processed with the PCA method for compression. In the next step, the two clustering algorithms are employed. The first Fuzzy-ART is responsible for determination of a number of clusters, while the second Fuzzy C-Means perform frame clustering based on the color histogram features. However, reported results indicate that using only color information is not enough to obtain the satisfactory results [7].

In the paper by Medentzidou and Kotropoulos, a video summarization method based on shot boundary detection with penalized contrast is proposed [36]. This method also relies on color analysis, using the HSV color space, however. The mean of the hue component is proposed as a way of change detection. Then, video segments are extracted to capture video content. These are then described with a linear model with the addition of noise. As reported, the method obtains results comparable to other methods, such as [2].

The other video summarization method, called VSCAN, was proposed by Mahmoud et al. [35]. Their method is based on a modified density-based spatial clustering of applications with noise-clustering method (DBSCAN), which is used to video summarization from color and texture features of each frame.

In our framework, we utilize tensor analysis. In the next section, we provide a brief introduction to this domain, introducing notation and concepts necessary for the understanding of the presented method. In the first row, the papers by de Lathauwer et al. can be recommended [30-32]. Tensors and their decompositions are also described in the papers by Kolda [27], Kolda and Bader [26], and also in the books, for example, by Cichocki [6], or the one by Cyganek [10].

In this paper, we also rely on the concepts of the data stream analysis, concept drift detection, as well as data classification in data streams. Recently, these gained much attention among researchers. This new domain of data processing is analyzed, for instance, in the book by Gama [17] or in the papers by Krawczyk et al. [28] or Woźniak et al. [53], to name a few. The two domains - that is,streams of tensor data-were pioneered by the works by Sun et al. [43, 44], as will be further discussed.

\section{Introduction to Tensors and Their Decompositions}

In this section, we present the basics of visual signal representations with tensors, as well as signal analysis based on tensor decompositions. Especially, tensor decompositions gained much attention, since they lead to discovery of latent factors, multidimensional data classification [9, 42] as well as for data compression [52]. Among many, the most prominent is the higher order singular value decomposition (HOSVD), canonical decomposition (Candecomp/Parafac) [25, 26, 51], as well as the Tucker [46] and the best rank- $\left(R_{1}, R_{2}, \ldots, R_{P}\right)$ decomposition [10, 11, 30, 32]. The method presented in this paper relies on the latter one, so its details as well as its computational aspects are discussed in further sections of this paper. 


\section{Signal Processing with Tensors}

Multidimensional measurements and signals are frequently encountered in science and industry. Examples are multimedia, video surveillance, hyperspectral images, sensor networks, to name a few. However, their processing with the standard vectorbased methods is usually not sufficient due to the loss of important information contained in internal structure and relations among data. Thus, for analysis and processing, more appropriate are methods that directly account for data dimensionality. In this respect, it was shown that tensor-based methods frequently offer many benefits, such as in the case of the tensorfaces, as well as view synthesis proposed by Vasilescu and Terzopoulos [48-50], or data dimensionality reduction by Wang and Ahuja [51, 52], handwritten digits recognition proposed by Savas and Eldén [42] or road signs recognition by Cyganek [9], to name a few. In this section, we present a brief introduction to the tensor analysis necessary for understanding of further parts of this paper. However, this short introduction by no means excerpts the subject. Further information can be found in many publications, such as, for instance, [5, 10, 11, 24, 25, 32, 48].

Let us now define a $P$-dimensional tensor as follows ${ }^{1}$ :

$$
\mathcal{A} \in \Re^{N_{1} \times N_{2} \times \ldots N_{P}},
$$

with each $k$ th dimension denoted by $N_{k}$ and for $1 \leq k \leq P$. Regardless of its physical meaning, a tensor can also be interpreted as a multidimensional cube of real-valued data, in which each dimension corresponds to a different component or measurement of the input data domain [10].

Based on the above definition, let us denote a $j$ th vector (called a fiber) of a $P$ thorder tensor $\mathcal{A}$ which is obtained by an exclusive change of one index $n j$ $\left(1 \leq n_{j} \leq N_{j}\right)$ while keeping all other indices fixed. A similar and very important concept is a tensor flattening (also called - matricization [25]). For a Pth-order tensor $\mathcal{A}$, its $j$ th flattening is defined as the following matrix:

$$
\mathbf{A}_{(j)} \in \Re^{N_{j} \times\left(N_{1} N_{2} \ldots N_{j-1} N_{j+1} \ldots N_{P}\right)} .
$$

A $j$ th flattening is obtained from $\mathcal{A}$ after selecting a $j$ th dimension, which becomes a row index of $\mathbf{A}_{(j)}$, whereas a product of all the rest indices constitutes a column index of $\mathbf{A}_{(j)}$. Columns of $\mathbf{A}_{(j)}$ are called $j$ th fibers of $\mathcal{A}$, and they span a $j$ th tensor subspace.

A $k$ th modal product $\mathcal{T} \times{ }_{k} \mathbf{M}$ of a tensor $\mathcal{T} \in \Re^{N_{1} \times N_{2} \times \ldots N_{P}}$ and a matrix $\mathbf{M} \in$ $\Re^{Q \times N_{k}}$ is a tensor $\mathcal{S} \in \Re^{N_{1} \times N_{2} \times \ldots N_{k-1} \times Q \times N_{k+1} \times \ldots N_{P}}$, whose elements are expressed as follows:

$$
\mathcal{S}_{n_{1} n_{2} \ldots n_{k-1} q n_{k+1} \ldots n_{P}}=\left(\mathcal{T} \times_{k} \mathbf{M}\right)_{n_{1} n_{2} \ldots n_{k-1} q n_{k+1} \ldots n_{P}}=\sum_{n_{k}=1}^{N_{k}} t_{n_{1} n_{2} \ldots n_{k-1} n_{k} n_{k+1} \ldots n_{P}} m_{q n_{k}} .
$$

\footnotetext{
1 Tensors are denoted with the calligraphic letters, vectors and matrices are in bold face.
} 
That is, Eq. (3) is an extension of the "classical" matrix multiplication, in which the product involves multiplication of elements of the $k$ th fibers of the tensor $\mathcal{T}$ and consecutive rows of the matrix $\mathbf{M}$. As a result, the $j$ th dimension of $\mathbf{T}$ is changed to $Q[10,30]$.

On the other hand and contrary to the well-known matrix algebra, there is no single definition of a rank of a tensor. There are at least three different concepts of a tensor rank from which the so-called $r$ th rank will be used throughout this paper: An $r$ th rank of a tensor $\mathcal{A}$ is a dimension of the vector space spanned by the columns of the $r$ th flattening $\mathbf{A}_{(r)}$ of this tensor.

\section{The Best Rank-( $\left.R_{1}, R_{2}, \ldots, R_{P}\right)$ Tensor Decompositions}

There are many types of tensor decompositions which aim at representing a tensor as a product of other tensors. These components frequently need to fulfill specific conditions such as orthogonality or sparsity. The goals can be manifold, such as to reveal latent components of an input tensor, to span orthogonal tensor subspaces, or for data reduction, to name a few $[5,6,10,24,30]$. The first decomposition method was proposed by Tucker in the mid-60s to analysis of psychometric data [46]. Recent 20 years benefited in the development of many new tensor decomposition methods, though [5, 6, 10, 26, 27]. Among them, usually, the following three are highlighted: the higher order singular value decomposition (HOSVD) [8, 9], the Candecomp/Parafac (CP) [25], as well as the best rank- $\left(R_{1}, R_{2}, \ldots, R_{P}\right)$ decomposition [32]. These extend the concept introduced by Tucker, as will be discussed.

A Tucker decomposition of the tensor $\mathcal{T} \in \Re^{N_{1} \times N_{2} \times \ldots \times N_{P}}$ is an approximating tensor $\tilde{\mathcal{T}}$ given as follows [46]:

$$
\tilde{\mathcal{T}}=\mathcal{Z} \times{ }_{1} \mathbf{S}_{1} \times{ }_{2} \mathbf{S}_{2} \ldots \times{ }_{P} \mathbf{S}_{P}
$$

and minimizing the following function:

$$
\Theta(\mathcal{T})=\|\mathcal{T}-\tilde{\mathcal{T}}\|_{F}^{2}
$$

where $\mathcal{Z}$ stands for a core tensor and $\mathbf{S}_{i} \in \Re^{N_{i} \times R_{i}}$ stand for the mode matrices.

After some basic algebraic operations, from (4), the following formula for the core tensor is obtained:

$$
\mathcal{Z}=\tilde{\mathcal{T}} \times{ }_{1} \mathbf{S}_{1}^{T} \times{ }_{2} \mathbf{S}_{2}^{T} \ldots \times_{P} \mathbf{S}_{P}^{T}
$$

Now, inserting (6) to (4) and then to (5) yields

$$
\Theta(\mathcal{T})=\left\|\mathcal{T}-\mathcal{T} \prod_{k=1}^{P} \times_{k}\left(\mathbf{S}_{k} \mathbf{S}_{k}^{T}\right)\right\|_{F}^{2}
$$

In other words, the Tucker decomposition given in (7) reads that a tensor $\mathcal{T}$ is approximated by its projection onto the space spanned by the matrices $\mathbf{S}_{k}$. Thus, the whole task is to compute the series of $\mathbf{S}_{k}$ mode matrices. However, in many 
applications, it is beneficial to request in (4) orthogonality or specific ranks of $\mathbf{S}_{k}$. If such a constraint is assumed, then the Tucker decomposition (4) leads to the best rank- $\left(R_{1}, R_{2}, \ldots, R_{P}\right)$ decomposition, defined as follows [32].

The best rank- $\left(R_{1}, R_{2}, \ldots, R_{P}\right)$ approximation of a tensor $\mathcal{T} \in \Re^{N_{1} \times N_{2} \times \ldots \times N_{P}}$ is a tensor $\tilde{\mathcal{T}}$ of ranks in each of its modes $\operatorname{rank}_{1} \tilde{\mathcal{T}}=R_{1}, \operatorname{rank}_{2} \tilde{\mathcal{T}}=R_{2}, \ldots$, $\operatorname{rank}_{P} \tilde{\mathcal{T}}=R_{P}$, respectively, which minimizes the function (7).

The best rank- $\left(R_{1}, R_{2}, \ldots, R_{P}\right)$ tensor decomposition is visualized in Fig. 3, whereas Algorithm 1 presents the basic steps of its computation. It solves the linear optimization problem by means of the Higher Order Orthogonal Iteration (HOOI) method [3, 26, 32]. It does optimization of only one matrix $\mathbf{S}_{i}$ in (4) per iteration while keeping all the other fixed. In the next iteration, the matrix $\mathbf{S}_{i+1}$ is chosen and the procedure continues until convergence conditions are met. Description of this algorithm with analysis of the computational complexity and its $\mathrm{C}++$ implementation can be found in the literature [10].

In Algorithm 1, the function $f d s(\mathbf{S}, R, \mathbf{T})$ denotes the fast dominating subspace computation. It serves computation of the $R$ left leading eigenvectors of the matrix $\mathbf{S}$, with the initial values taken from the matrix T. For this purpose, the classical SVD Jacobi-based algorithm can be used. However, in this work, we propose to use a much faster fixed-point algorithm with further modifications, as discussed in Sect. 4.3. This is a key modification to the best $\operatorname{rank}-\left(R_{1}, R_{2}, \ldots, R_{P}\right)$ algorithm which allows for more efficient processing of the video streams.

However, in the presented system, the dynamic tensor analysis will be used which aims at processing a series of tensors, to build their representing model. For this purpose, Algorithm 1 needs to be extended, as will be discussed.

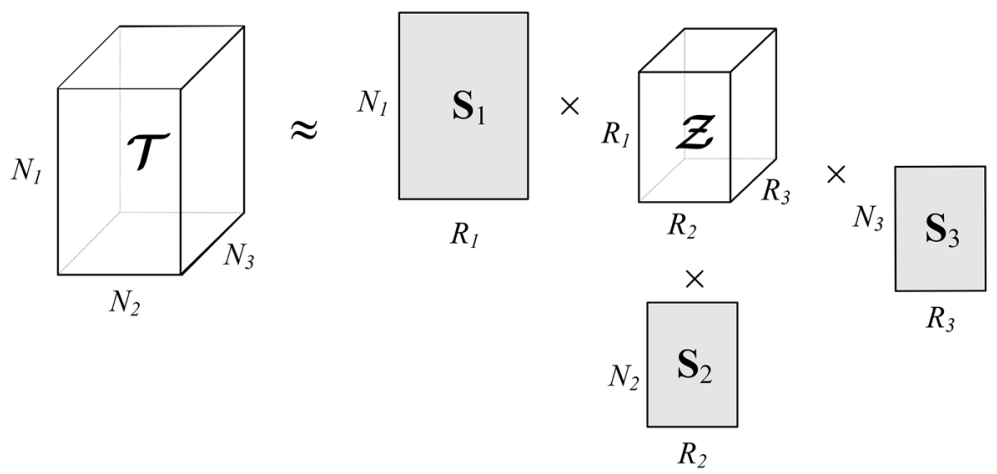

Fig. 3 Visualization of the best rank- $\left(R_{1}, R_{2}, \ldots, R_{P}\right)$ tensor decomposition 


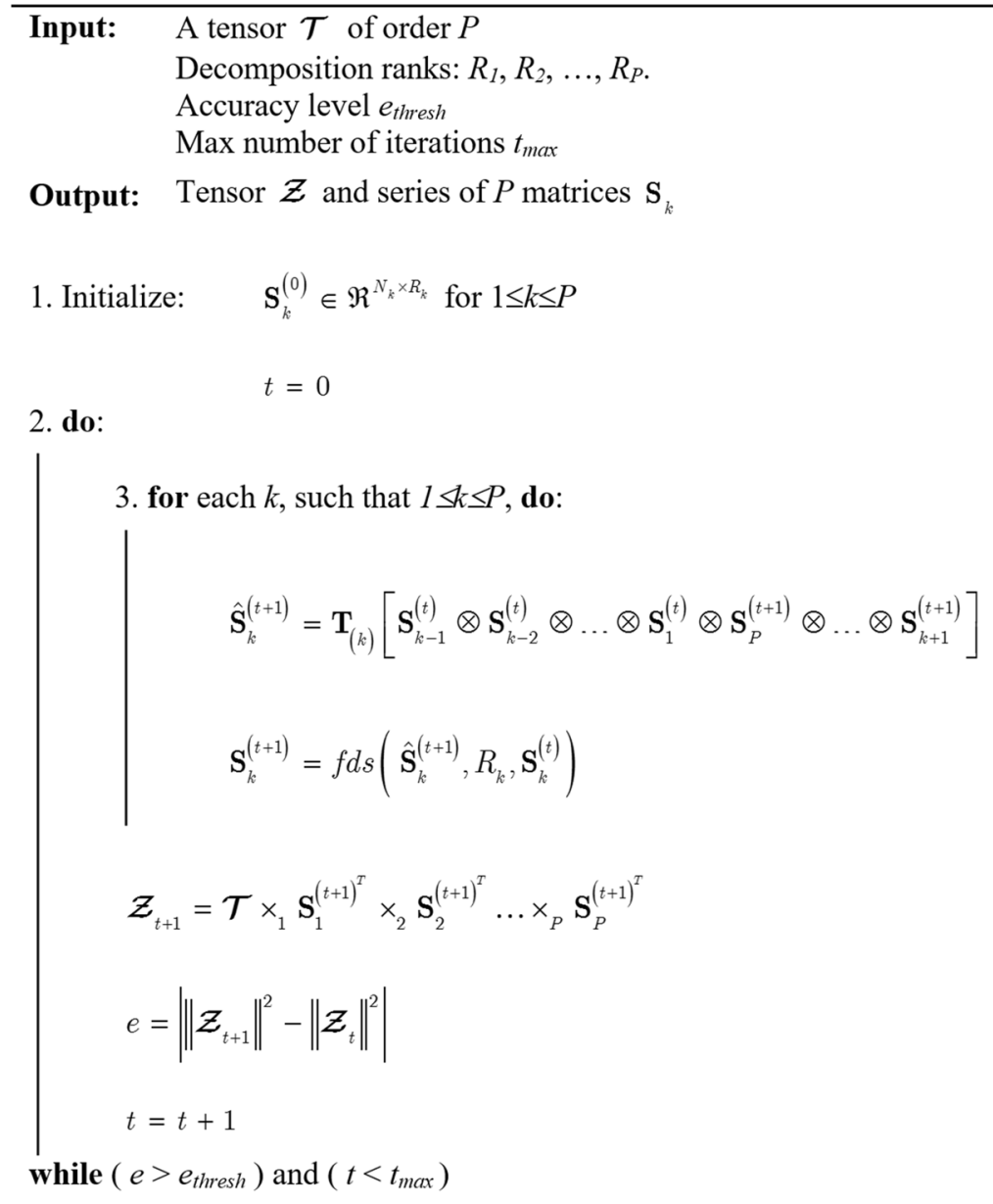

4. Store $\mathcal{Z}$ and the series of the mode matrices $\mathbf{S}_{k}$.

Algorithm 1. The best rank- $\left(R_{1}, R_{2}, \ldots, R_{P}\right)$ tensor decomposition algorithm.

\section{Tensor Model and Inferring Method for Video Data Stream Analysis}

Presented video shot detection method employs the stream and dynamic tensor analysis methods proposed by Sun et al. [43, 44]. However, we introduced a number of modifications to allow its efficient operation on video streams of different 
dimensions, as well as a new method of construction of a tensor model specific to the video signals. In this section, we describe the basic ideas behind the tensor stream processing framework, as well as our proposed features which allow efficient video shot detection in various types of video streams.

\section{System Architecture and Basic Concepts of the Tensor Stream Analysis}

In the proposed system, a stream of input tensor data of the same order $P$ is assumed. As already mentioned, in this version, we do not assume any feature extraction from the input tensors. This means that any type of signal can be applied to the presented algorithms. Thus, the method works fine with both the monochrome as well as color video streams. This makes the proposed method versatile. Nevertheless, if there is additional knowledge of a signal type, then methods that compute specific features can be more discriminative but only for this specific type of a signal. This happens with color histograms, frequently used in video shot detection $[2,7,35]$. Such methods will not work with monochrome or hyperspectral images, though. Thus, in our framework, features can also be included in the input tensors to make the signals more discriminative, but this possibility is left for further research. To proceed, we introduce a number of definition after the work of Sun et al. [44]: a sequence of tensors is a series of $m$ tenors $\mathcal{A}_{i}$, where $1 \leq i \leq m$, each of

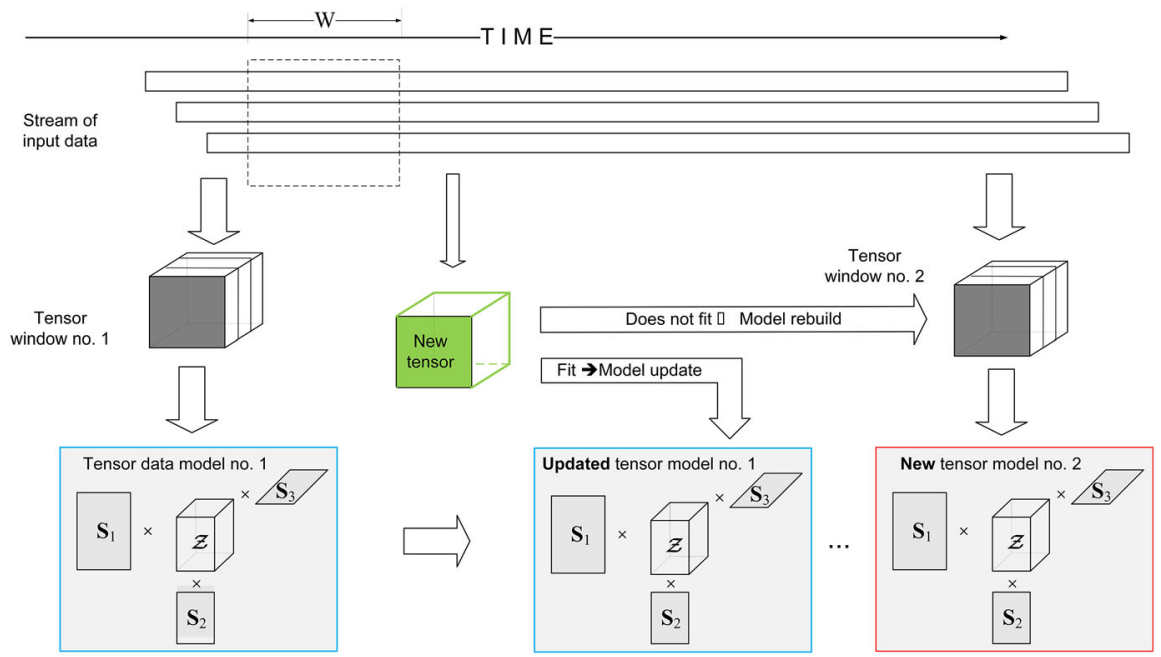

Fig. 4 Architecture of the system for processing a stream of tensor data. In the input stream, a window of fixed size $W$ is selected. From this, a tensor model is built with a modified best rank- $\left(R_{1}, R_{2}, \ldots, R_{P}\right)$ tensor decomposition. The other tensors in the stream are checked to fit to the model. If a concept drift is detected at a given time stamp, then a new model is built from a next tensor window $W$, starting from that time stamp. Otherwise, a model is only updated 
$P$ th-order $\mathcal{A} \in \Re^{N_{1} \times N_{2} \times \ldots N_{P}}$, and assuming that $m$ is constant. A stream of tensors is a sequence of $m$ tensors, where $m$ is a natural value increasing with time.

Our proposed system operates as follows. From the input stream of tensor data, a window of consecutive frame tensors of size $W$ is selected. All of them are used to build a best rank- $\left(R_{1}, R_{2}, \ldots, R_{P}\right)$ tensor model, as described in Algorithm 1. However, the main modification of this model, which we incorporate after the work by Sun et al., consists of computing a covariance matrix out of all flattened versions of the tensors from the selected window $W$. This way, for each of the $P$ flattenings, a single covariance matrix is created from all tensors in the input window. Thus, such a covariance matrix conveys statistical information on all of the input tensors in a given flattening mode. The next computational perk of this approach is that the covariance matrix is a positive definite one for which a more effective eigenvalue decomposition method could be applied, as will be discussed. It is worth noticing that such an approach differs from the building and decomposing of a new large tensor composed of all tensors from the window stream after adding a new dimension $N_{P+1}$ of value $W$. Details of the modified best rank- $\left(R_{1}, R_{2}, \ldots, R_{P}\right)$ decomposition, as well as of the model build-and-update procedures from the streams of tensor data are presented in the next section.

Figure 4 shows architecture of the system for processing a stream of tensor data. As already mentioned, in the input stream, a window of fixed size $W$ is selected. From this, a tensor model is built with a modified best rank- $\left(R_{1}, R_{2}, \ldots, R_{P}\right)$ tensor decomposition. The other tensors in the stream are checked if they fit to the model form the latest window $W$ based on a special fitness function, as will be discussed. If a concept drift in the stream is detected, then a new model is built from a next tensor window $W$ at the given time stamp. Otherwise, the model needs only to be updated to account for the new tensor. This is the model adaptation mechanism. Both the model build-and-update algorithms are described in the next sections.

\section{A Modified Best Rank- $\left(R_{1}, R_{2}, \ldots, R_{P}\right)$ Tensor Decomposition for Stream Tensors}

Algorithm 2 presents the best rank- $\left(R_{1}, R_{2}, \ldots, R_{P}\right)$ tensor decomposition algorithm modified for processing of a stream of tensor data. 


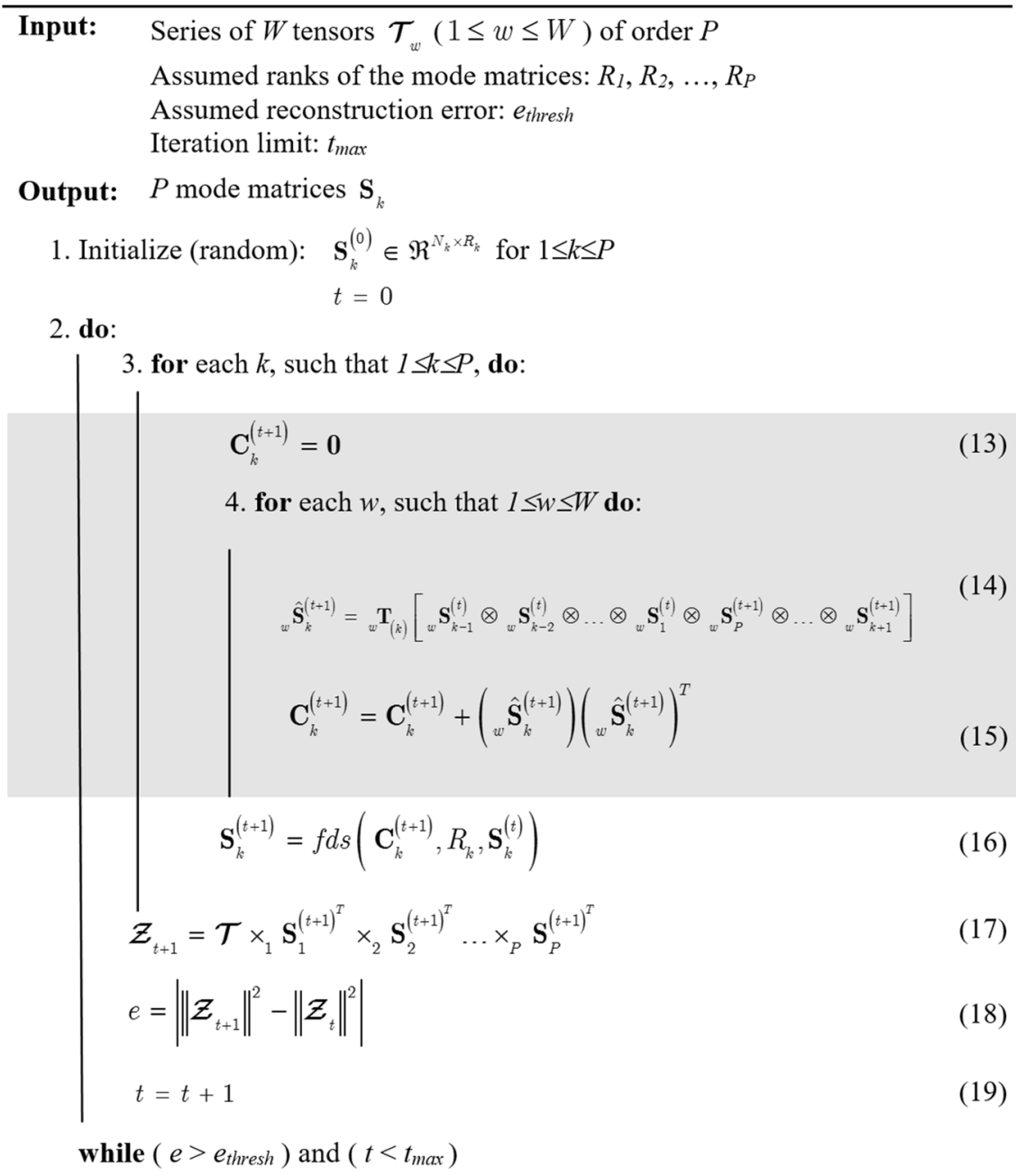

5. Store the last values of the matrices $\mathbf{S}_{k}$.

Algorithm 2. The best rank- $\left(R_{1}, R_{2}, \ldots, R_{P}\right)$ tensor decomposition algorithm adjusted to processing streams of tensor data. Compared to the best rank- $\left(R_{1}, R_{2}, \ldots, R_{P}\right)$ for a single tensor in Algorithm 1, the main modification lies in computation of the variance matrices out of each of the flattenings of the series of input tensors.

When compared to Algorithm 1, the new steps are (14) and (15) which serve computations of the covariance matrices from each tensor in the input window $W$ and at each of the $P$ flattening modes. In step (16), the fast dominating subspace is computed with the $f d s\left(\mathbf{C}_{k}^{(t+1)}, R_{k}, \mathbf{S}_{k}^{(t)}\right)$ function. Its details are described in the next step. Nevertheless, it is worth noticing that in each iteration, it starts with an initial value of $\mathbf{S}_{k}^{(t)}$ computed in the previous step, thus speeding up the convergence, as was verified experimentally. Only the first iteration starts with a random 
initialization. It is important to notice that the two loops in Algorithm 2 determine the speed of a model build and, in consequence, computation of the whole method.

As already mentioned, after building the model from the input tensor stream, the incoming tensor data are checked to fit that model. Therefore, a suitable fitness function needs to be developed. This, in the context of video stream analysis, will be discussed in the next section. If a new tensor does not fit to the model, it needs to be rebuilt from scratch, starting at the position in the stream of a just checked tensor. On the other hand, if it fits, then the model also needs to be rebuilt to account for this new tensor and to adjust to the changes in the stream. However, despite the speed-up of the model build procedure presented in Algorithm 2 its continuous recomputation at each tensor in the stream is computationally not tractable. Therefore, instead, a mode update procedure is employed, as proposed by Sun et al. [43]. It simply relies on an update of the covariance matrices in (15) in Algorithm 2 in each of the flattening modes with the new tensor data. Such a procedure is well known in the statistics and relies on the following computation:

$$
C_{k}^{t+1}=\alpha C_{k}^{t}+S_{k}^{t+1} S_{k}^{t+1^{T}},
$$

where $\alpha$ is a forgetting factor of a previous model, and $\mathbf{S}_{k}^{(t+1)}$ is a $k$ th mode matrix of a new (updating) tensor at a time step $t+1$. Summing up, the described method assumes model build from which only the covariance mode matrices need to be stored. Thus, the input tensors from the window $W$ are not accessed anymore which results in significant memory savings. Then, on a new tensor, if decided to update the model, for each of the flattening modes, the proper covariance matrices of a model are updated in accordance with (20). Finally, $f d s\left(\mathbf{C}_{k}^{(t+1)}, R_{k}, \mathbf{S}_{k}^{(t)}\right)$ from the step (16) in Algorithm 2, needs to be computed, but only once. However, it should be pointed out that the covariance matrix update rule (15), as well as (20), holds under assumption of the same mean value among data. If this is not true, then to keep the model accurate enough, also the mean updating component should be added to the aforementioned equations [34]. Nevertheless, we also follow this simplifying assumption and use the updating model (20). This is justified by the fact that we are interested in detecting signal changes which, if happen, usually also lead to a change of the mean values in data. In such a case, the model will not hold anymore which will trigger its rebuild process, as discussed in Sect. 4.5, and the shot will be detected.

\section{Efficient Computation of the Dominating Tensor Subspace}

Application of the tensor decomposition to analysis of tensor streams, such as video signals, would be very limited or even useless if cannot be efficiently computed. In this respect, the best would be to have a real-time response or at least an answer in a user acceptable time, which certainly is a subjective notion. To be more concrete, we measure processing time in terms of the input (tensor) frame processing. However, efficiency means also computational stability, as will be discussed. 


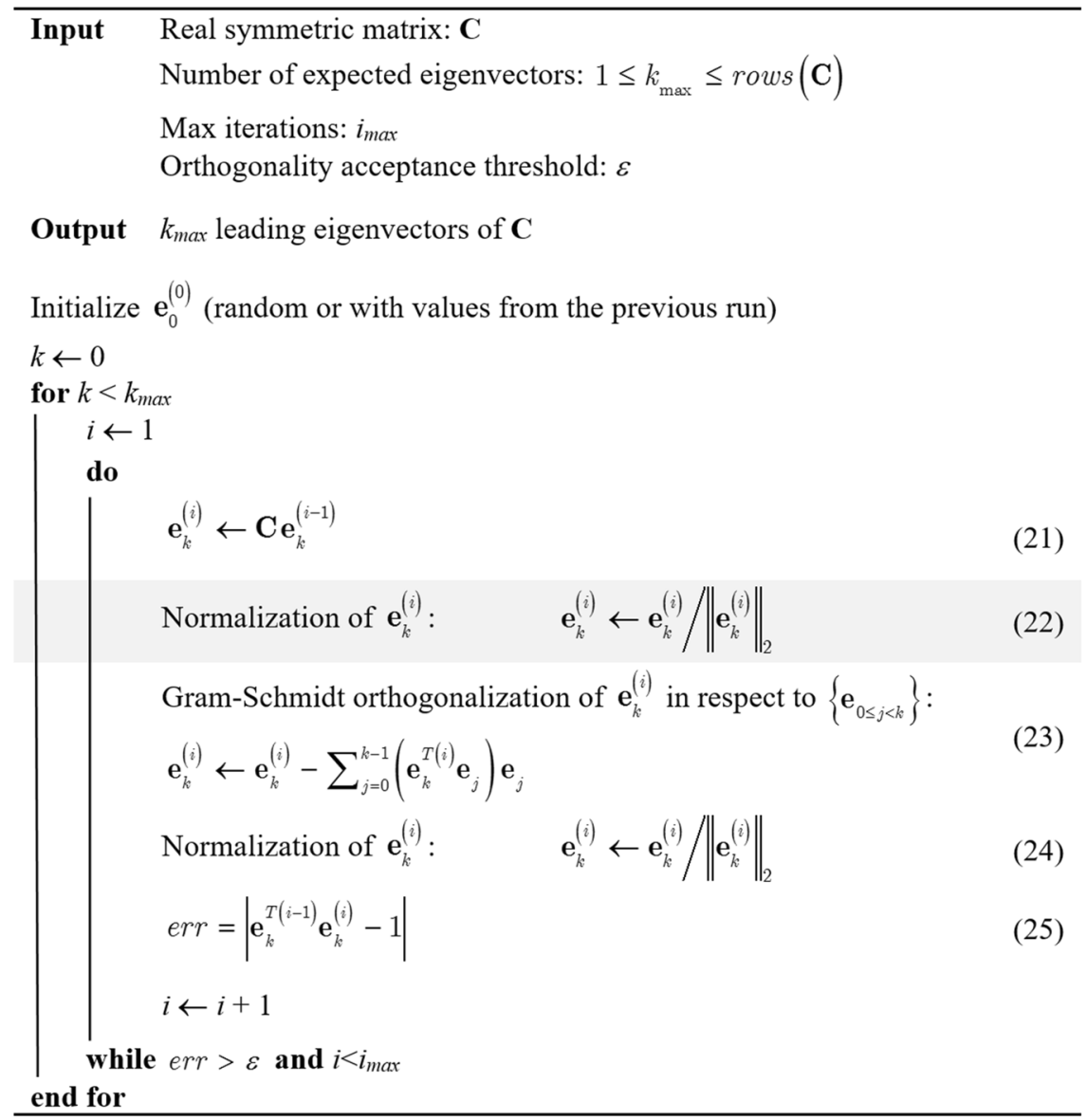

Algorithm 3. The fixed-point method of computations of the leading eigenvectors of the real symmetric matrix $\mathbf{C}$. The proposed additional normalization (22) improves the numerical stability of the algorithm.

As already mentioned, thanks to the formulation of computation steps in Algorithm 2 with only the positive real symmetric matrices, it is possible to employ a faster algorithm than the SVD decomposition of matrices for a general case. For this purpose, in our system, the so-called fixed-point eigen decomposition algorithm is used for computation of the $f d s$ function from the step (16) in Algorithm 2. This method was first proposed to be used in tensor decomposition by Muti and Bourennane [39]. Then, it was also used for computation of the HOSVD tensor decomposition in the work by Cyganek and Woźniak [12]. Algorithm 3 shows the key steps of this method.

However, after longer use of this algorithm for different tensor decompositions, we noticed its problems with its numerical stability. Under closer examination, it 
appeared that the problem is usually caused by the multiplication step (21) in Algorithm 3, especially if elements of the input matrix $\mathbf{C}$ have magnitudes few order of magnitude higher than values of the vector $\mathbf{e}_{k}$. Therefore, our proposition is to add an additional normalization step (22) just after the multiplication (21) and before the Gram-Schmidt orthogonalization step (23). Our experiments showed that the proposed modification improved the numerical stability of the whole procedure with insignificant additional computations.

The next modification proposed in this paper is an automatic mechanism of evaluating the number of important eigenvalues $k_{\text {imp }}$, which is determined dynamically during the run of the algorithm. The main idea consists in checking a ratio of logarithms of the eigenvalues, corresponding to the consecutive eigenvectors, respectively. If this ratio exceeds a preset threshold, in our experiments set to 1.5 , then the procedure is stopped. In practice, this simple rule allows for significant computational savings.

The next simple modification is initialization of the starting values of the eigenvectors with these values computed in the previous iteration, rather than initialization with random values, as presented in (16). This relies on a simple observation that the computed eigenvectors do not lie far from each other between consecutive iterations. Therefore, such initialization, usually, leads to the faster convergence than when started from the randomly chosen initial values. Random initialization is applied only at the first run.

\section{Concept Drift Detection in Video Tensor Streams}

The goal of the concept drift detection is to discover conditions in which a data model is not sufficient to well represent content of the current data stream. This usually happens due to changes of the statistical properties of the data stream caused by external factors. The concept drift detection in different streams belongs to a dynamical research area $[17,18,28]$. In the case of video streams, a concept drift usually corresponds to content change in video signal due to a hard or soft shots, as already discussed.

In the proposed system, the data model is obtained with the best rank- $\left(R_{1}\right.$, $\left.R_{2}, \ldots, R_{P}\right)$ tensor decomposition, as already described. The model is stored as a series of matrices $\mathbf{S}_{k}$ and the variance matrices $\mathbf{C}_{k}$. Then, a model fit measure for a new tensor $\mathcal{X}$ is indirectly obtained from the error value (7). However, we need to develop a measure of checking if a new tensor $\mathcal{X}$ fits to the model.

The simplest way to build a fitness algorithm is first, to check consistency of a model and then relate the model consistency to the fit measure of an unknown tensor data $\mathcal{X}$. This can be done by computing all values of $\Theta\left(\mathcal{T}_{i}\right)$ in (7), for all $\mathcal{T}_{i}$ belonging to the window $W$. Then, their mean and standard deviation can be used to describe the model [43]. These are simply given as follows:

$$
\bar{\Theta}=\frac{1}{\# W} \sum_{i=1}^{w} \Theta_{i},
$$

and 


$$
\sigma^{2}=\frac{1}{\# W-1} \sum_{i=1}^{w}\left(\Theta_{i}-\bar{\Theta}\right)^{2},
$$

where $\# W$ denotes a number of tensors in the window $W$. Finally, a new tensor, $\mathcal{X}$, can be checked to fit to the model by a simple $\pm 3 \sigma$ rule as follows:

$$
\left\|\Theta_{\mathcal{X}}-\bar{\Theta}\right\|<3 \sigma .
$$

However, after examining this simple model on a number of video streams, we noticed that, instead of the absolute error values $\Theta$, better results are obtained if the differences $\Delta \Theta$ of $\Theta$ are used. The error function difference is defined simply as

$$
\Delta \Theta_{i} \equiv \Theta_{i-1}-\Theta_{i}
$$

Finally, the fitness measure has to be further modified to account for the series of tensor frames which change insignificantly or do not change at all. This happens, for instance, for the series of frames from a video with no content change. In such cases, the $\sigma$ parameter tends toward 0 , and in a result, even not significant change in a new frame would not fulfill in accordance with (28), and in a consequence, the entirely new model rebuild process would be launched, which is not justified and computational costly. Thus, to account for such situations, our fitness measure is expressed as follows:

$$
\left\|\Delta \Theta_{\mathcal{X}}-\bar{\Theta}_{\Delta}\right\|<a \sigma_{\Delta}+b
$$

where $\bar{\Theta}_{\Delta}$ and $\sigma_{\Delta}$ are the mean and standard deviation computed from the differences of consecutive fit values in (29), respectively, $a$ is multiplicative factor (set in our experiments to the range 1.5-3.7), and $b$ is an additive constant (in our experiments set to $0.2-2.5$ ).

\section{Video Tensor Stream Model Build-and-Update Scheme}

All of the aforementioned mechanisms are assembled here into a complete tensorbased method for efficient video stream analysis. Algorithm 4 shows our proposed tensor model build and concept drift detection mechanisms for video stream analysis.

It is worth noticing that the initial rank values $R_{1}, R_{2}, \ldots, R_{P}$ are the maximal possible ranks that are considered when building the model. However, real ranks are determined based on the already described automatic rank assessment mechanism and, in practice, are usually much smaller than the conservatively assumed initial ranks. In our experiments, these are set as a percentage of the dimensions of the tensor frames. For instance, these are $R_{1}=0.25 N_{1}, R_{2}=0.25 N_{2}$, and $R_{3}=N_{3}$, where $N_{1}$ and $N_{2}$ stand for the column and row dimensions, whereas $N_{3}$ for the color one, respectively.

The next stabilizing mechanism introduced in our system is a counter of consecutive frames that do not fit the current tensor model. If the model is not fulfilled for all consecutive $G$ frames, then only the total model rebuild procedure is 
launched. This saves from costly model rebuild procedure in case of spurious frames, e.g., caused by mechanical deterioration of the celluloid film material, scratches, noise, etc. In our system, $G$ was in the ranges $1-7$.

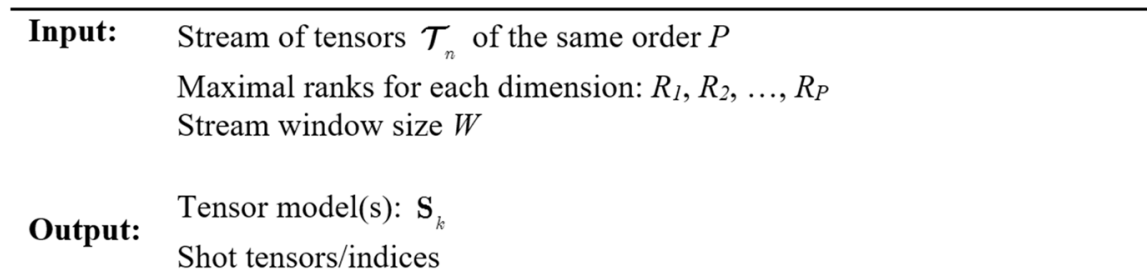

1. Build a tensor model $\mathbf{S}_{k}$ in the window $W$ (Algorithm 2);

2. for each stream tensor

3. Compute parameters (26)-(28) for (29);

4. if model fit measure (30) does NOT hold for $G$ consecutive frames

5. Model rebuild (Algorithm 2) from current position and shot detection (shifted back by $G$ );

else

6. Model update in accordance with (20);

Algorithm 4. The tensor model build and concept drift detection mechanisms for video streams analysis.

Last but not least, problem is how to update the parameters (26)-(28) when updating the model with a new tensor frame. In this case, the mean and the standard deviations are recomputed after obliterating a value of the first tensor from the previous model $W$. Thus, for efficient computation, all $\Theta\left(\mathcal{T}_{i}\right)$ need to be stored for each $i$ th tensor of the model. Upon model update with a $\mathcal{T}_{i+1}$ tensor, also its new value $\Theta\left(\mathcal{T}_{i+1}\right)$ is computed and added to the stored series. Finally, the mean and standard deviation are recomputed in accordance with (26)-(28), all for differences of error functions (29), as already discussed.

\section{Experimental Results}

The method was implemented in $\mathrm{C}++$ in the Microsoft Visual 2015 IDE. The DeRecLib library was used for tensor processing and basic decompositions [10, 13]. The experiments were run on a computer equipped with the Intel ${ }^{\circledR}$ Xeon ${ }^{\circledR}$ E-1545 
processor operating at $2.9 \mathrm{GHz}$, with $64 \mathrm{~GB}$ RAM, and 64-bit version of Windows 10.

For evaluation, we used the VSUMM database and provided therein summarization of different videos $[2,22]$. Such an approach has been undertaken by many researchers and allows qualitative evaluation and comparison among a group of video summarization methods. This database contains 50 videos from the OpenVideo Project [21]. The videos are stored in the MPEG-1 format with $30 \mathrm{fps}$ and resolution $352 \times 240$ pixels, color and sound, with duration in the range of 1-4 min. They are classified into different genres, such as documentary, educational, ephemeral, historical, and lecture.

The key control parameters of the proposed method are contained in Table 1. These are described and discussed in the previous sections. There are also some auxiliary parameters, such as a maximum number of iterations in $t_{\max }$ in Algorithm 2 or the orthogonality threshold $\varepsilon$ in Algorithm 3, but results are not very sensitive to a particular choice of their values. These, once set as already described, remained fixed in all experiments.

From the parameters collected in Table 1, the most sensitive is the values $a$ and $b$ of the tensor frame fit measure in (30), as well as the model window size $W$ and the model forgetting factor $\alpha$ in (20). On the other hand, the assumed ranks constitute only the initial values for the tensor best rank- $\left(R_{1}, R_{2}, \ldots, R_{P}\right)$ decomposition, since the real number of important factors is computed in each decomposition based on the eigenvalue ratio method, as described in Sect. 4.3.

For the qualitative evaluation of performance of the method, we incorporated the methodology, as well as ground truth user summaries, in the VSUMM method, proposed by de Avila et al. [2, 22], as well as the VSCAN method presented in the work of Mahmoud et al. [23, 35]. In the former approach, the Comparison of User Summaries (CUS) measure is evaluated. It consists of comparing two sets of the keyframes, one build manually by a user (a ground truth) and the second obtained with a tested method. Certainly, this requires a method of comparing two frames, as well as a threshold to determine if the frames are, or are not, similar. For this purpose, de Avila et al. propose two parameters: $C U S_{A}=n_{A U} / n_{U}$ and

Table 1 Key control parameters of the presented method

\begin{tabular}{|c|c|c|}
\hline Parameter & Description & $\begin{array}{l}\text { Values used in the } \\
\text { experiments }\end{array}$ \\
\hline$W$ & $\begin{array}{l}\text { Size of the tensor-frame window used to build a model (step } 4 \\
\text { in Algorithm 2) }\end{array}$ & $3-55$ \\
\hline$\alpha$ & A model forgetting factor in (20) & $0.6-1.0$ \\
\hline$a, b$ & Tensor-frame fit measure (30) & $\begin{array}{l}a \text { in } 1.5-3.7 \\
b \text { in } 0.2-2.5\end{array}$ \\
\hline$G$ & $\begin{array}{l}\text { Number of consecutive frames to launch rebuild of the tensor } \\
\text { model (31) }\end{array}$ & $1-11$ \\
\hline $\begin{array}{l}R_{1}, R_{2}, \ldots \\
\quad R_{P}\end{array}$ & Assumed ranks of the mode matrices (Algorithm 2) & $\begin{array}{l}5-75 \% \text { of each dimension } \\
\text { of the tensor frame }\end{array}$ \\
\hline
\end{tabular}


$C U S_{E}=\sim n_{A U} / n_{U}$, where $n_{A U}$ denotes a number of matching keyframes from the automatic summary $(A S)$ and the user-annotated summary, $\sim n_{A U}$ is the complement of this set (i.e., the frames that were not matched), whereas $n_{U}$ is a total number of keyframes from the user summary only (US). However, as presented by Mahmoud et al., more informative parameters are precision $P$ and recall $R$, since they also convey information on the keyframes present in $A S$ and not present in $U S$ or vice versa. As a trade-off of the two, the so-called $F$ measure is used [20, 35, 45]. Thus, in our experiments, we used the measures defined as follows:

$$
P=\frac{n_{A U}}{n_{A}}, R=\frac{n_{A U}}{n_{U}}, F=2 \frac{P R}{P+R},
$$

where $n_{A U}$ denotes a number of keyframes from the automatic summary that match the ones from the user's summary, and $n_{A}$ is a number of total keyframes from the automatic summary only while $n_{U}$ from the user's summary.

As already mentioned, the comparisons are done between the found shots and the user-annotated ones from the VSUMM database. Figure 5 shows an example of the user summary obtained for the Drift Ice as a Geologic Agent, segment 8 in the VSUMM repository. However, for different users, their summaries also differ in a number of selected keyframes, as well as particular frames chosen for this purpose [2].

Figure 6 depicts shots found by our method in the Drift Ice as a Geologic Agent, segment 8 test video. In this case, the color video was converted to monochrome for speed-up. The model windows was set to $W=11$, the model check parameters $(a$, $b)=(3.0,2.0)$, and $G=3$ (Table 1). However, during the experiments, we observed that it is possible to select hard shots from all of the detected shots by a simple thresholding, since for these types of shots, the fitness function is much larger. It is due to a measure of differences instead of absolute values of the fitness of the frames to the model. In Fig. 6, hard cuts detected with this method are shown in red, while the model fit values are in black. Comparing the user summary from Fig. 5 with the detected shots, shown in Fig. 7 and in the plot in Fig. 6, we easily obtain that $P=9 / 13 \approx 0.69, R=9 / 10=0.9$, what yields $F=0.78$ for this case.

What we have noticed is that the results depend on the chosen model window size $W$, but its value is not critical. That is, for many videos, good results are obtained for $W$ in a certain range, such as 7-15 in this case, rather than for a particular value. This happens, because the model is continuously updated in accordance with the procedure, as described in Algorithm 4. In addition, a choice of
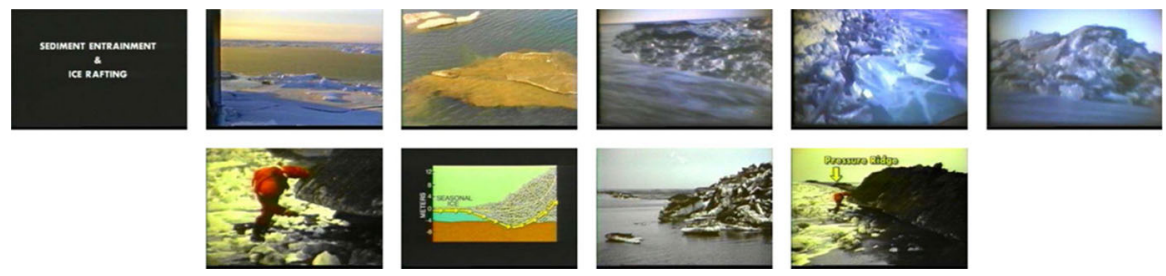

Fig. 5 Example of the user summary created for the Drift Ice as a Geologic Agent, segment 8 in the VSUMM repository 


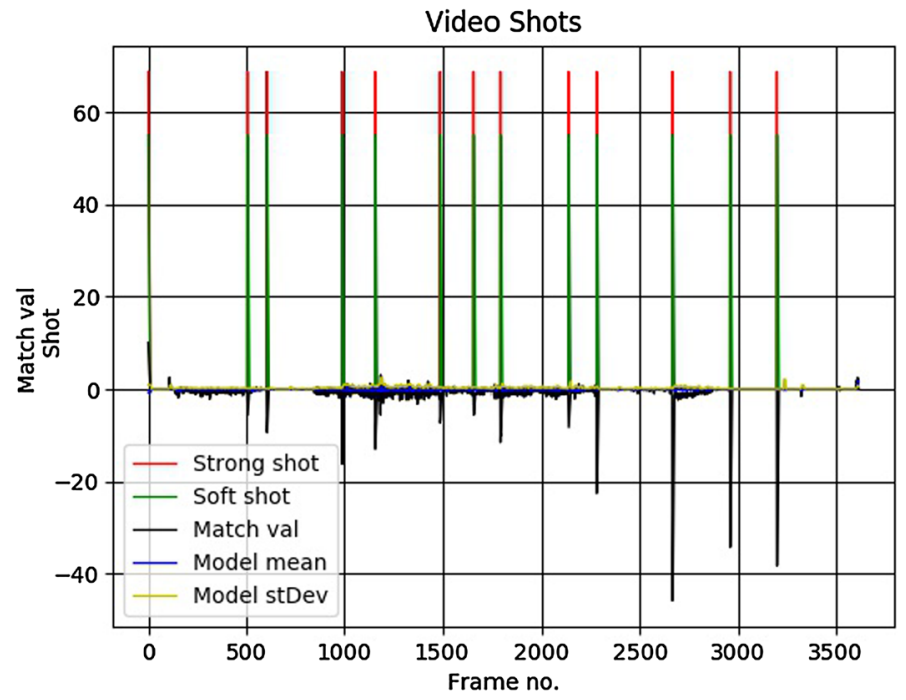

Fig. 6 Video shots found by our method in the Drift Ice as a Geologic Agent, segment 8 test video. Used parameters: monochrome video, $W=11,(a, b)=(3.0,2.0), G=3$ (see Table 1). Hard cuts denoted in red. Model fit value shown in black
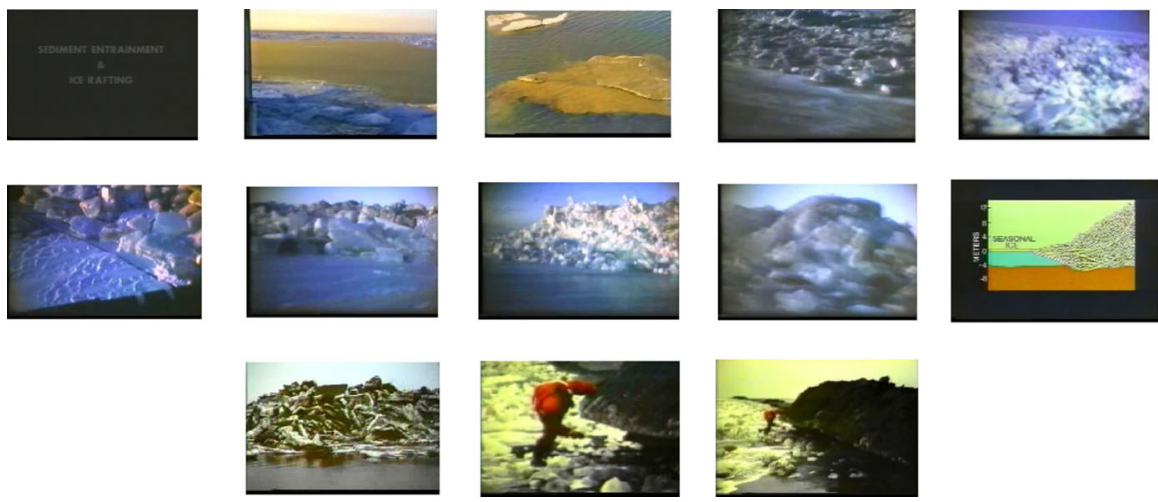

Fig. 7 Keyframes of the strong shots found by the proposed method in the Drift Ice as a Geologic Agent, segment 8 test video [21]. These are indicated by the red bars in Fig. 6

the parameter $G$, controlling the series of consecutive "not-fit" frames, is not a critical one. We set this value to 3 to avoid shot detection on some spurious but single frames. Such situation are usually due to some deterioration of a particular frame, e.g., due to scratches or dust if it was stored on the celluloid film tape, etc.

Figure 8 depicts frame shots in larger context, as detected in the experiment which results are shown in Fig. 6.

However, the results depend much on the parameters $a$ and $b$ of the fit function (30). Especially, this choice determines how many soft shots will be detected. For example, in the experiment which results are shown in Fig. 6, these were set to ( $a$, 

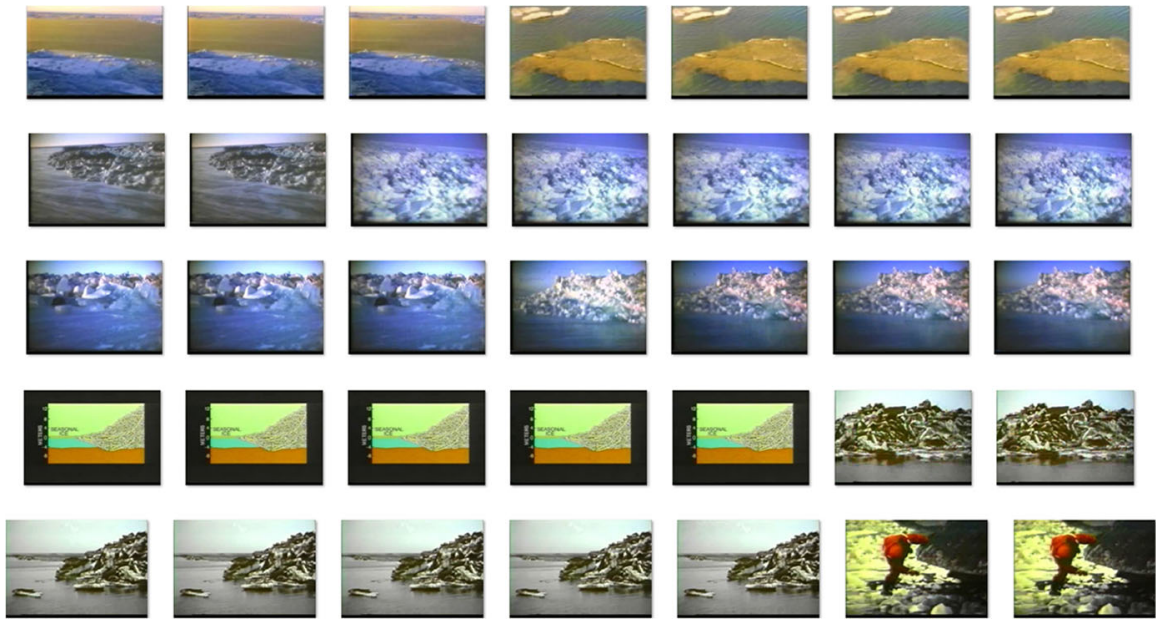

Fig. 8 Examples of the detected shots computed by the proposed method and shown in Figs. 6 and 7 (from top): frames 602-608, 1155-1161, 2660-2666, and 2954-2960

$b)=(3.0,2.0)$ which rather excludes some of the fade-in and fade-out types of shots.

Figure 9 shows results of two experiments on the monochrome and color versions of the Drift Ice as a Geologic Agent, segment 8 test video. However, in this case, we set the parameters $(a, b)$ to $(3.0,2.0)$. This resulted in detection of more frames that did not fit to the local tensor model, and these were reported as detected shots.

Choice of the window size $W$ used to build a tensor model in some degree depends on the content of the video. However, in our experiments, it was possible to select a range of values $7-25$ which produced acceptable results. When compared performance of the method in respect to window size $W$, we noticed that short windows, of $W$ in the range 3-6, result in detection of many soft shots, even if such are caused by small change in the consecutive frames. Figure 10 shows shot detection results for $W=5$ and all other parameters exactly the same as in the experiment, as shown in Fig. 9b. On the other hand, long windows of $W$ in the range over 30, which correspond to the video time laps over $1 \mathrm{~s}$, result in soft shots to be omitted by the model. This happens due to the model rebuild procedure (20) which makes it updated with each new frame which fitted to that model. This procedure relies on the model forgetting parameter $\alpha$ set in our experiments in the range 0.6-1.0.

Figure 11 shows detected video shots found by our method in the Ice Wallaw test video. Used parameters are: monochrome video, $W=7, G=7,(a, b)=(3.7,0.2)$. Detected shots are shown with green bars. Among these, red bars denote strong shots.

Figure 12 shows video shots found in the Exotic Terrane (segment 04) test video. In both cases, the same model window is used of $W=11$. However, the top experiment was set for detection of exclusively hard shots by setting the parameters 

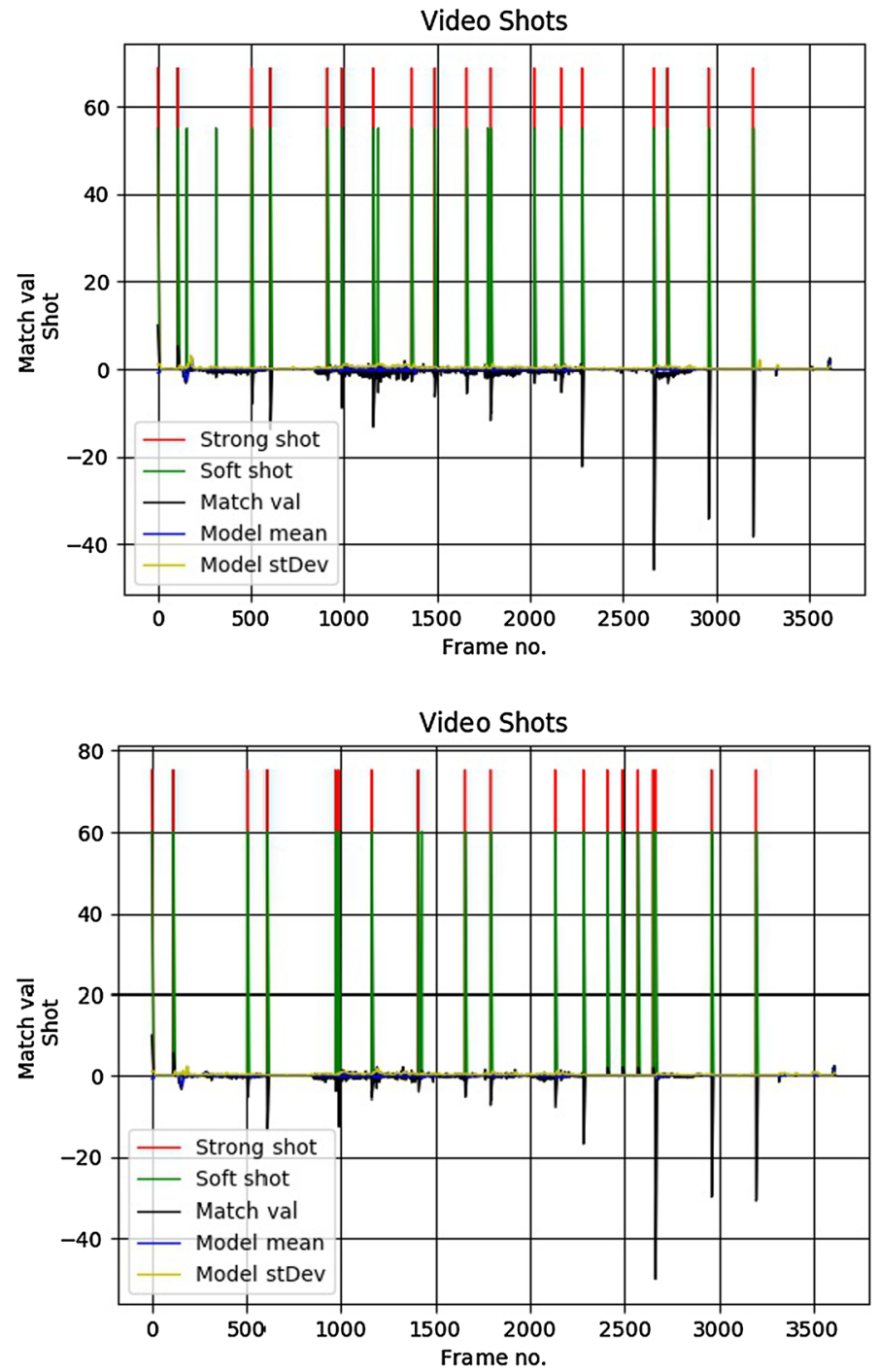

Fig. 9 Video shots found by our method in the Drift Ice as a Geologic Agent, segment 8 test video. Used parameters: $W=11,(a, b)=(3.7,0.8)$. Top monochrome video, bottom color video

$(a, b)=(3.0,2.0)$. On the other hand, the lower plot corresponds to settings $(a$, $b)=(3.7,0.2)$ which also allow for detection of soft shots.

As already mentioned, the method was tested with the user-annotated database of the VSUMM method by de Avila et al. [2, 22], and compared to the results obtained with other methods such as OV [37], DT [38], STIMO [16], as well as VSCAN by Mahmoud et al. [35]. Table 2 contains averaged $F$ values of the obtained results of these methods and the tensor method proposed in this paper. The former was obtained from the webpage [23]. For both the color and monochrome videos, 


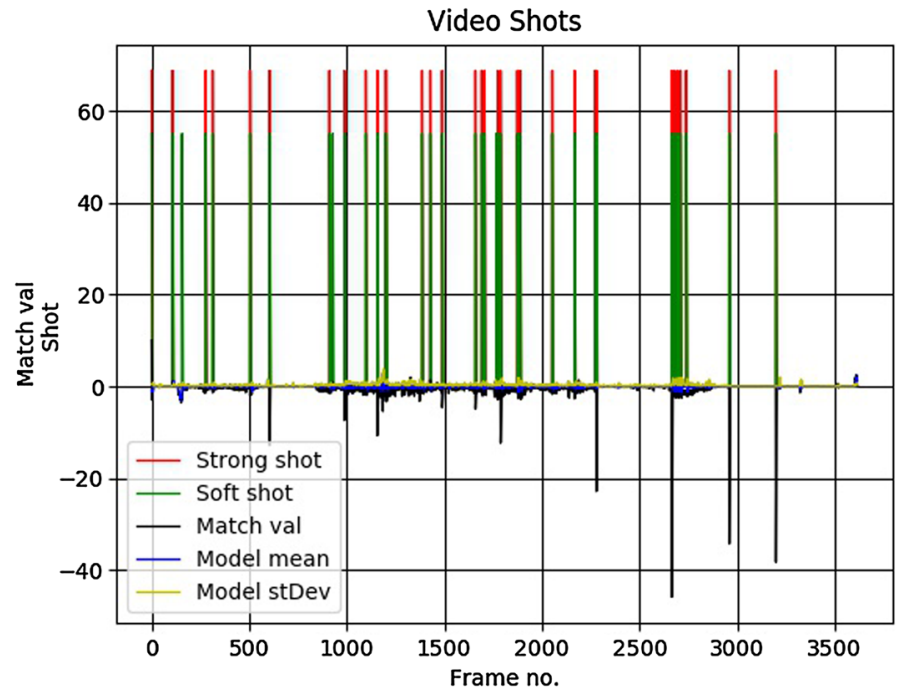

Fig. 10 Video shots found by our method in the Drift Ice as a Geologic Agent, segment 8 test video. Used parameters: color video, $W=5,(a, b)=(3.7,0.8)$

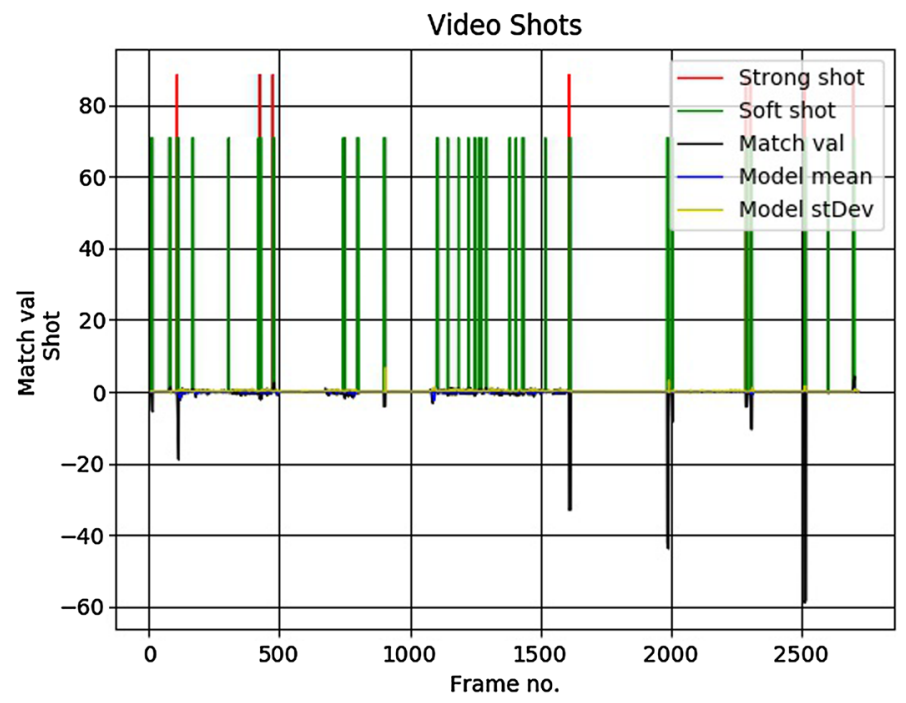

Fig. 11 Video shots found by our method in the Ice Wallaw test video. Used parameters: monochrome video, $W=7, G=7,(a, b)=(3.7,0.2)$. Detected shots shown with green bars. Among these, red bars denote strong shots

respectively, the same parameters were used as follows: $W=13, a=2.0, b=1.0$ $\alpha=0.97$, and $G=9$, and the initial rank values were $R_{1}=0.1, R_{2}=0.1$, and $R_{3}=1.0$ (only for color) times each dimension of each tensor frame. Thus, for a frame of $352 \times 240 \times 3$, this sets the initial maximal ranks to $35 \times 24 \times 3$, 

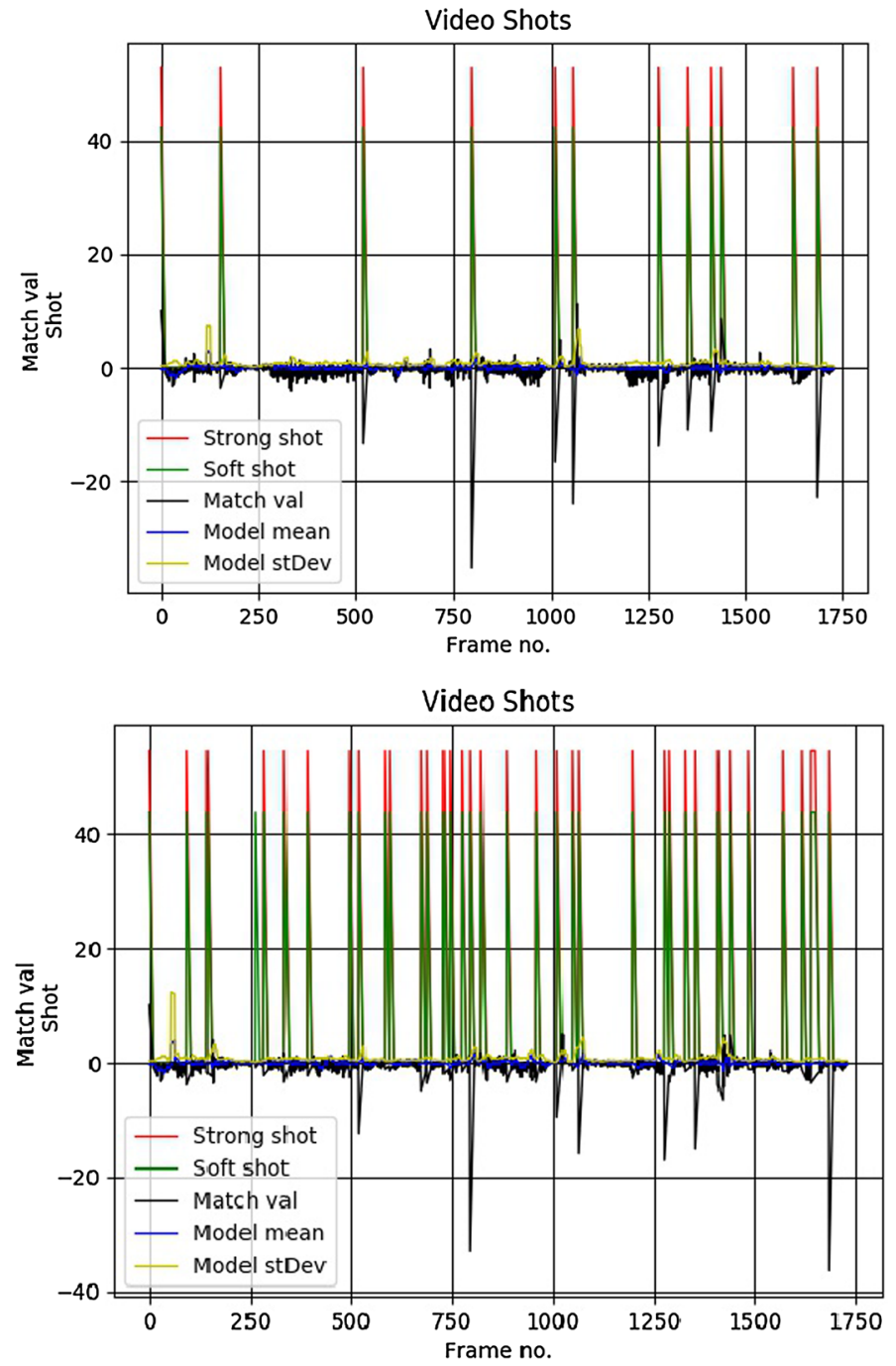

Fig. 12 Video shots found by our method in the Exotic Terrane (segment 04) test video. Used parameters: $W=11,(a, b)=(3.0,2.0)$-top, $(a, b)=(3.7,0.2)$-bottom. Top monochrome video, bottom color video

respectively. However, as already mentioned, these are then adapted in the runtime to the current signal conditions based on the automatic rank selection method. Detailed values obtained for each of the test frames, for both the color and monochrome versions, are available from the Internet. ${ }^{2}$

We see that the proposed method performs comparatively or better than other methods, except for the VSCAN. It can be considered as good results taking into consideration that the method does not compute any specific features. The

\footnotetext{
2 http://home.agh.edu.pl/ cyganek/TensorVideoNGC2017.mht.
} 
Table 2 Mean $F$ values for different methods. Last column shows average results obtained with the presented method for the color and monochrome videos, respectively

\begin{tabular}{lllllll}
\hline Method & OV & DT & STIMO & $\begin{array}{l}\text { VSUMM } \\
{[2]}\end{array}$ & $\begin{array}{l}\text { VSCAN } \\
{[35]}\end{array}$ & $\begin{array}{l}\text { Tensor (this } \\
\text { paper) }\end{array}$ \\
\hline $\begin{array}{l}\text { F-measure color } \\
\text { video }\end{array}$ & 0.67 & 0.61 & 0.65 & 0.72 & 0.77 & $\mathbf{0 . 7 3}$ \\
$\begin{array}{l}\text { F-measure } \\
\text { monochrome }\end{array}$ & - & - & - & - & - & $\mathbf{0 . 7 0}$ \\
\hline
\end{tabular}

experiments showed that a difference between the color and monochrome video is about 0.03 . After closer analysis, we noticed that using color leads to removal of some of the false positives. Nevertheless, the difference in accuracy between the color and monochrome versions is not too big, which can be explained by a small value of the color dimension, that is three, as well as by a correlation between the color channels. On the other hand, VSUMM and VSCAN rely on color histograms. This means that the proposed method can work with the monochrome videos, whereas the former cannot. In addition, the proposed method can almost automatically accommodate another dimensions, such as in the case of multiview or hyperspectral streams. When analyzing results of operation of the proposed method on various test video streams, we observed that frequently, it tends to detect more shots than in the user-annotated surveys used for methods evaluation (false positives). This depends strongly on the chosen parameters, especially the ones controlling the frame fitness measure, as was discussed. Nevertheless, detected shots can also be considered as valid, since usually, there is a noticeable change of frame contents, as can be observed. However, such operation results in high recall parameter but rather poor precision at the same time, what explains results, as presented in Table 2.

The average execution times are presented in Table 3. These were measured over all run tests for monochrome, as well as color video streams. As can be seen, the change from the 2D tensor for the monochrome case, to the 3D for color, changes around five time longer execution time. In this case, there are three time larger data streams. However, the real reason is a significant change in computation of the tensor model in computation of the Kronecker product in (14). Examining this formula, we notice that the number of the multiplied matrices is $P-1$. This means that for the $2 \mathrm{D}$ tensors, there is nothing to multiply and the matrix is returned untouched, whereas for 3D tensors, the product needs to be computed and returned. We will investigate further this problem to achieve a speed-up.

Table 3 Average execution time for the monochrome 2D-tensor and color 3D-tensor streams

\begin{tabular}{lll}
\hline & $\begin{array}{l}\text { Monochrome video } \\
(2 \mathrm{D}) 352 \times 240\end{array}$ & $\begin{array}{l}\text { Color video (3D) } \\
352 \times 240\end{array}$ \\
\hline \multirow{2}{*}{ Frames } & 1948 & 1948 \\
& 15.6 & 3.2 \\
\hline
\end{tabular}




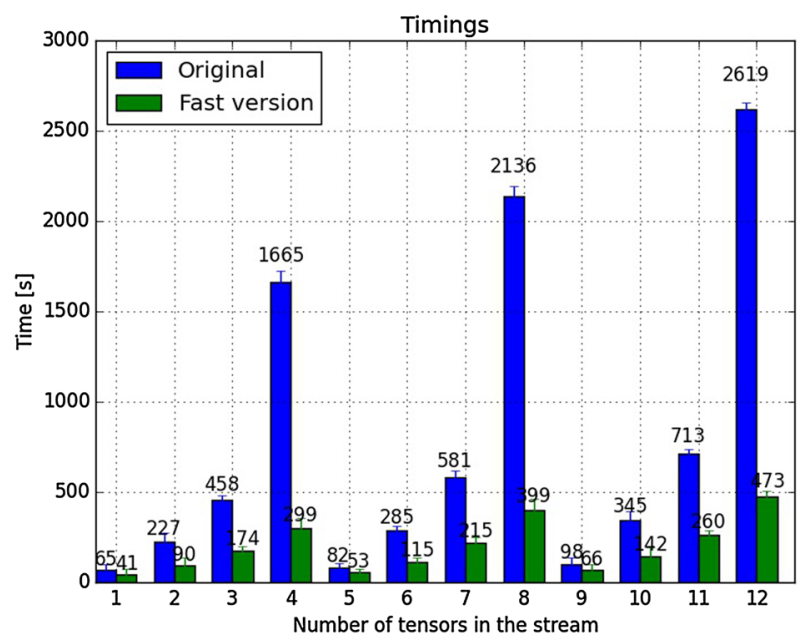

Fig. 13 Comparison of execution times for the standard SVD vs. fixed-point computation of the eigenvalues for different groups of test tensor streams

Finally, we measured time speed-up caused by application of the faster method for computation of the dominating tensor subspaces, as discussed in Sect. 4.3. Figure 13 shows comparison of execution time for the standard SVD method, compared to the modified fixed-point algorithm used in the proposed framework. In this experiment, four groups of 3D tensors were first created, each of dimensions $3 \times 128$ (nos. 1, 5, and 9), $3 \times 256$ (nos. 2, 6, 10), $3 \times 384($ nos. 3, 7, 11), as well as $3 \times 512$ (nos. 4, 8, and 12). Then, 12 streams were created, starting from 1 tensor up to 12 in the stream. Time measurements in our software framework are depicted in Fig. 13. From this, it is evident that especially for larger tensors and longer streams, the speed-up ratio reaches five times in benefit of the proposed method.

It is worth noticing that in time-demanding applications, the method can be further speeded up by processing each $n$th frame from the stream. Such strategy is used in some of the reported methods. However, in the presented framework, we did not use this option and all the tests were run with no frame decimation.

\section{Conclusions}

In this paper, the tensor-based method for video stream analysis and concept drift detection is presented. The method builds a tensor model from a number of frames from the input stream. The model is then continuously updated by the frames in the stream if they fit to the model based on the proposed fitness function. However, if for a number of consecutive frames in the stream, the fitness function indicates their high deviation from the model, the model is rebuilt starting from the current position. We proposed an efficient model construction method which is based on the best rank- $\left(R_{1}, R_{2}, \ldots, R_{P}\right)$ tensor decomposition for processing of streams of tensor data. 
The method was built and tested in the framework of video stream processing for detection of the video shots. The main benefit of using the tensor representation and decomposition is their natural ability to account for many internal dimensions of the frames constituting a video stream as well as its ability to operate with original signals with no necessity of feature detection. This can be beneficial in the case of multidimensional signals or measurements coming in a stream and for which their statistical properties are not known a priori. Nevertheless, features can be easily incorporated as the next dimensions of the input tensors. However, each additional dimension incurs a polynomial grow of the computational complexity, as well as memory consumption. The analysis of tensors joining signal and/or additional signal features is left for further research.

The method was adjusted to work with color and monochrome videos by designing a specific concept drift detector. The method was then tested on the OpenVideo database with monochrome and color versions of the test videos. For both types, the achieved accuracy can be compared favorably with majority of the video keyframe detection methods, especially designed to process exclusively color videos. Hence, the proposed tensor method has the ability to work with any type of video signals, such as the monochrome, color, hyperspectral, etc. with no a priori assumption on specific statistical properties of signals. On the other hand, we noticed relatively poor recognition of specific types of the soft temporal signal changes. This happens because if a change is not sufficiently abrupt to cause model rebuild, the adaptively progressing model gets gradually adapted to the slow/soft signal changes. However, these can be monitored by, e.g., higher standard deviation of the fit measure, although its level depends on the signal type. In addition, a better concept drift detector method can be designed which is also left for future research.

Nevertheless, the method requires significant memory and computational resources. To improve its performance, the fast method of dominant tensor subspace construction is proposed. Thanks to this, a significant speed-up ratio was obtained when compared to the standard eigen problem solution based on the SVD decomposition. The tensor subspace method was further modified, as proposed in this paper, to increase its numerical stability and to allow for automatic determination of the dominating eigenvalues.

Further research will be oriented toward improvement of accuracy and speed of the proposed method, possibly by developing more efficient model fitness functions, as well as by development of parallel tensor decomposition methods.

Acknowledgements This work was supported by the Polish National Science Center NCN under the Grant No. 2014/15/B/ST6/00609. This work was supported by the statutory funds of the Department of Systems and Computer Networks, Faculty of Electronics, Wroclaw University of Science and Technology.

Open Access This article is distributed under the terms of the Creative Commons Attribution 4.0 International License (http://creativecommons.org/licenses/by/4.0/), which permits unrestricted use, distribution, and reproduction in any medium, provided you give appropriate credit to the original author(s) and the source, provide a link to the Creative Commons license, and indicate if changes were made. 


\section{References}

1. Asghar, M.N., Hussain, F., Manton, R.: Video indexing: a survey. Int. J. Comput. Inf. Technol. 03(01), 148-169 (2014)

2. de Avila, S.E.F., Lopes, A.P.B., da Luz Jr., A., Araújo, A.A.: VSUMM: a mechanism designed to produce static video summaries and a novel evaluation method. Pattern Recogn. Lett. 32, 56-68 (2011)

3. Bader, B.W., Kolda, T. G.: Algorithm 862: MATLAB tensor classes for fast algorithm prototyping. ACM Trans. Math. Softw. 32(4), 635-653 (2006)

4. Bellman, R.E.: Adaptive control processes: a guided tour. Princeton University, Princeton (1961)

5. Cichocki, A., Zdunek, R., Amari, S.: Nonnegative matrix and tensor factorization. IEEE Signal Process. Mag. 25(1), 142-145 (2008)

6. Cichocki, A., Zdunek, R., Phan, A.H., Amari, S.-I.: Nonnegative matrix and tensor factorizations. Applications to Exploratory Multi-way Data Analysis and Blind Source Separation. Wiley, Hoboken (2009)

7. Cayllahua-Cahuina, E.J.Y., Cámara-Chávez, G., Menotti, D.: A static video summarization approach with automatic shot detection using color histograms. In: Proceedings of the International Conference on Image Processing, Computer Vision, and Pattern Recognition (IPCV). The Steering Committee of The World Congress in Computer Science, Computer Engineering and Applied Computing (WorldComp), pp. 1-12 (2012)

8. Cyganek, B., Krawczyk, B., Woźniak, M.: Multidimensional data classification with chordal distance based kernel and support vector machines. Eng. Appl. Artif. Intell. 46(A), 10-22 (2015)

9. Cyganek, B.: An analysis of the road signs classification based on the higher-order singular value decomposition of the deformable pattern tensors. Advanced Concepts for Intelligent Vision Systems ACIVS 2010, LNCS 6475, pp. 191-202. Springer, Berlin (2010)

10. Cyganek, B.: Object detection and recognition in digital images. Theory and Practice. Wiley, Hoboken (2013)

11. Cyganek, B.: Object recognition with the higher-order singular value decomposition of the multidimensional prototype tensors. In: 3rd Computer Science On-line Conference (CSOC 2014). Advances in Intelligent Systems and Computing. Springer, Berlin, pp. 395-405 (2014)

12. Cyganek, B., Woźniak, M.: On robust computation of tensor classifiers based on the higher-order singular value decomposition. In: The 5th Computer Science On-line Conference on Software Engineering Perspectives and Application in Intelligent Systems 2016 (CSOC2016). Advances in Intelligent Systems and Computing, vol. 465, pp. 193-201. Springer, Berlin (2016)

13. DeRecLib, http://www.wiley.com/go/cyganekobject. Accessed 29 July 2017

14. FabroDel, M., Böszörmenyi L.: State-of-the-art and future challenges in video scene detection: a survey. Multimedia Systems, vol. 19, Issue 5, pp 427-454, Springer, Berlin (2013)

15. Fu, Y., Guo, Y., Zhu, Y., Liu, F., Song, C., Zhou, Z.-H.: Multi-view video summarization. IEEE Trans. Multimedia 12(7), 717-729 (2010)

16. Furini, M., Geraci, F., Montangero, M., Pellegrini, M.: STIMO: STIll and moving video storyboard for the web scenario. Multimed Tools Appl 46(1), 47-69 (2010)

17. Gama, J.: Knowledge Discovery from Data Streams. CRC Press, Boca Raton (2010)

18. Gama, J., Žliobaite I., Bifet, A., Pechenizkiy, M., Bouchachia, A.: A survey on concept drift adaptation, ACM Computing Surveys (CSUR), Vol. 46, No. 4, pp. 44:1-44:37 (2014)

19. Gao, Y., Wang, W.-B., Yong, J.-H., Gu, H.-J.: Dynamic video summarization using two-level redundancy detection, Multimedia Tools and Applications, pp. 233-250 (2009)

20. Guan G, Wang Z, Yu K, Mei S, He M, Feng D.: Video summarization with global and local features. In: Proceedings of the 2012 IEEE International Conference on Multimedia and Expo Workshops, IEEE Computer Society, Washington, DC, pp. 570-575, 2012

21. The Open Video Project, https://open-video.org/. Accessed 29 July 2017

22. VSUMM, https://sites.google.com/site/vsummsite/home. Accessed 29 July 2017

23. VSCAN, https://sites.google.com/site/vscansite/home. Accessed 29 July 2017

24. Kay, D.: Schaum's Outline of Tensor Calculus. McGraw-Hill (1988)

25. Kiers, H.A.L.: Towards a standardized notation and terminology in multiway analysis. J. Chemom. 14, 105-122 (2000)

26. Kolda, T.G., Bader, B.W.: Tensor decompositions and applications. SIAM Review 51(3), 455-500 (2009)

27. Kolda, T.G.: Orthogonal tensor decompositions. SIAM J. Matrix Anal. Appl. 23(1), 243-255 (2001)

28. Krawczyk, B., Minku, L.L., Gama, J., Stefanowski, J., Woźniak, M.: Ensemble learning for data stream analysis: a survey. Inf. Fusion. 37, 132-156 (2017) 
29. Kuanar, S.K.: Video key frame extraction through dynamic Delaunay clustering with a structural constraint. J. Vis. Commun. Image Represent. 24(7), 1212-1227 (2013)

30. Lathauwer, de L.: Signal processing based on multilinear algebra. Ph.D. dissertation, Katholieke Universiteit Leuven (1997)

31. de Lathauwer, L., de Moor, B., Vandewalle, J.: A multilinear singular value decomposition. SIAM J Matrix Anal. Appl. 21(4), 1253-1278 (2000)

32. de Lathauwer, L., de Moor, B., Vandewalle, J.: On the best rank-1 and rank- $\left(R_{1}, R_{2}, \ldots, R_{\mathrm{N}}\right)$ approximation of higher-order tensors. SIAM J. Matrix Anal. Appl. 21(4), 1324-1342 (2000)

33. Lee, H., Yu, J., Im, Y., Gil, J.-M., Park, D.: A unified scheme of shot boundary detection and anchor shot detection in news video story parsing. Multimedia Tools \& Applications. 51, 1127-1145 (2011)

34. Li, Y.: On incremental and robust subspace learning. Pattern Recogn. 37, 1509-1518 (2004)

35. Mahmoud, K.A., Ismail, M.A., Ghanem, N.M.: VSCAN: an enhanced video summarization using density-based spatial clustering. Image analysis and processing-ICIAP 2013, vol. 1, pp. 733-742. LNCS Springer, Berlin (2013)

36. Medentzidou, P., Kotropoulos, C.: Video summarization based on shot boundary detection with penalized contrasts. In: IEEE 9th international symposium on image and signal processing and analysis (ISPA), pp. 199-203 (2015)

37. DeMenthon, D., Kobla, V., Doermann, D.: Video summarization by curve simplification. In: Proceedings of the sixth ACM international conference on Multimedia, ACM, pp. 211-218 (1998)

38. Mundur, P., Rao, Y., Yesha, Y.: Keyframe-based video summarization using Delaunay clustering. Internat. J. Dig. Libr. 6(2), 219-232 (2006)

39. Muti, D., Bourennane, S.: Survey on tensor signal algebraic filtering. Signal Process. 87, 237-249 (2007)

40. Ou, S.-H., Lee, C.-H., Somayazulu, V.S., Chen, Y.-K., Chien, S.-Y.: On-line multi-view video summarization for wireless video sensor network. IEEE J. Sel. Topics Signal Process. 9(1), 165-179 (2015)

41. Porwik, P., Orczyk, T., Lewandowski, M., et al.: Feature projection k-NN classifier model for imbalanced and incomplete medical data. Biocybern Biomed Eng 36(4), 644-656 (2016)

42. Savas, B., Eldén, L.: Handwritten digit classification using higher order singular value decomposition. Pattern Recogn. 40, 993-1003 (2007)

43. Sun, J., Tao, D., Faloutsos, C.: Beyond streams and graphs: dynamic tensor analysis. KDD’06, Philadelphia, Pennsylvania, USA (2006)

44. Sun, J., Tao, D., Faloutsos, C.: Incremental tensor analysis: theory and applications. ACM Trans. Knowl. Discov. Data 2(3), 11:1-11:37 (2008)

45. Truong, B.T., Venkatesh, S.: Video abstraction: a systematic review and classification. ACM Trans. Multimed. Comput. Comm. Appl. 3(1), 1-37 (2007)

46. Tucker, L.R.: Some mathematical notes on three-mode factor analysis. Psychometrika 31, 279-311 (1966)

47. Valdes, V., Martinez, J.: Efficient video summarization and retrieval tools. International Workshop on Content-Based Multimedia Indexing, pp. 43-48 (2011)

48. Vasilescu, M.A., Terzopoulos, D.: Multilinear analysis of image ensembles: TensorFaces. In: Proceedings of European Conference on Computer Vision, pp. 447-460 (2002)

49. Vasilescu, M.A., Terzopoulos, D.: Multilinear independent component analysis. In: IEEE Conference on Computer Vision and Pattern Recognition, CVPR 2005, Vol. 1, pp. 547-553 (2005)

50. Vasilescu, M.A., Terzopoulos, D.: Multilinear (Tensor) image synthesis, analysis, and recognitioin. IEEE Signal Processing Magazine, pp. 118-123 (2007)

51. Wang, H., Ahuja, N.: Compact Representation of Multidimensional Data Using Tensor Rank-One Decomposition. In: Proceedings of the 17th International Conference on Pattern Recognition, Vol. 1, pp. 44-47 (2004)

52. Wang, H., Ahuja, N.: A tensor approximation approach to dimensionality reduction. Int. J. Comput. Vision 76(3), 217-229 (2008)

53. Woźniak, M., Graña, M., Corchado, E.: A survey of multiple classifier systems as hybrid systems. Inf. Fusion 16, 3-17 (2014)

54. Wu, Z., Xie W., Yu J.: Fuzzy C-means clustering algorithm based on kernel method. In: Fifth International Conference on Computational Intelligence and Multimedia Applications (ICCIMA'03), pp. 1-6 (2003)

55. Zimek, A., Schubert, E., Kriegel, H.-P.: A survey on unsupervised outlier detection in high-dimensional numerical data. Stat. Anal. Data Min. 5(5), 363-387 (2012) 